\title{
AVALIAÇÃO ESPECTRAL E TEMPORAL DE REMANESCENTES DA MATA ATLÂNTICA COM DADOS SPOT-VGT E VARIÁVEIS METEOROLÓGICAS
}

\author{
SPECTRAL AND TEMPORAL EVALUATION OF ATLANTIC FOREST REMANESCENTS WITH \\ SPOT-VGT DATA AND METEOROLOGICAL VARIABLES
}

\author{
Pâmela Suélen Käfer ${ }^{1}$, Franciel Eduardo Rex ${ }^{2}$ \\ ${ }^{1}$ Universidade Federal do Rio Grande do Sul, Porto Alegre, Rio Grande do Sul, Brasil - \\ pamelaskafer@gmail.com \\ ${ }^{2}$ Universidade Federal do Paraná, Curitiba, Paraná, Brasil -francielrexx@gmail.com
}

RESUMO

\begin{abstract}
A Mata Atlântica é um dos biomas mais ricos do mundo em termos de biodiversidade e endemismo. Nesse sentido, a cidade de Porto Alegre é uma das capitais com maior porcentagem de remanescentes naturais do bioma, o que atesta a necessidade de entendimento e monitoramento da dinâmica dessas áreas florestais. Considerando que as ações de conservação e proteção destas áreas são grandes desafios, este trabalho objetivou analisar a dinâmica espectral e temporal de remanescentes florestais do bioma Mata Atlântica, na cidade de Porto Alegre, RS. Para isto, foram adquiridas séries temporais de Normalized Difference Vegetation Index (NDVI) provenientes de imagens de 10 dias do SPOT Vegetation, no período de 2012-2014. Além dos dados de NDVI, dados meteorológicos de temperatura e precipitação foram utilizados para complementar as análises. Foi observado que o padrão de comportamento da vegetação apresentou um ciclo definido nos dados decendiais de NDVI e este ciclo está claramente associado com as variações nas variáveis climáticas temperatura e precipitação. A variável temperatura mostrou-se mais correlacionada com os dados de NDVI do que a precipitação, principalmente pela capacidade de resiliência dos remanescentes florestais a períodos curtos de déficit hídrico. As variações nos dados temporais de NDVI podem ajudar a compreender o efeito de fatores climáticos sobre a vegetação local, podendo assim auxiliar no planejamento e mitigação de grande escala.
\end{abstract}

PALAVRAS-CHAVE: Dinâmica florestal, Índices espectrais, NDVI, Sensoriamento Remoto, Vegetação.

\begin{abstract}
The Atlantic forest is one of the richest biomes in the world in terms of biodiversity and endemism. In this sense, the Porto Alegre city is one of the capitals with the highest percentage of natural remnants of the biome, which attests the necessity of understanding and monitoring the dynamics of these forest areas. Considering that the conservation and protection actions of these areas are challenges, this study aimed to analyze the spectral and temporal dynamics of forest remnants of the Atlantic forest biome, in Porto Alegre city. For this purpose, NDVI time series from 10-day SPOT Vegetation images were acquired in the period from 2012-2014. In addition to the NDVI data, temperature and rainfall data were used to complement the analyzes. It was observed that the pattern of vegetation behavior showed a defined cycle in the decendial NDVI data and this cycle is clearly associated to variations in the climatic variables temperature and rainfall. The temperature variable was more correlated with the NDVI data than the rainfall, mainly due to the resilience of forest remnants to short periods of water deficit. Variations in the NDVI temporal data may help to understand the effect of climatic factors on local vegetation, thus helping in large scale planning and mitigation.
\end{abstract}

KEYWORDS: Forest dynamics, Spectral indices, NDVI, Remote sensing, Vegetation. 


\section{INTRODUÇÃO}

O bioma Mata Atlântica é considerado um dos biomas mais ricos do mundo em termos de biodiversidade e endemismo. Ao mesmo tempo é um dos mais ameaçados, estando em situação crítica de alteração de seus ecossistemas naturais (FUNDAÇÃO SOS MATA ATLÂNTICA, 2014; CYSNEIROS et al., 2016; TEIXEIRA et al., 2019). Apesar de a Mata Atlântica possuir uma legislação específica de proteção, todos os anos perde grandes áreas por conta de fatores como a expansão da agricultura, pecuária (COSTA \& FUTEMMA, 2006; SILVA et al., 2014) e urbana. Essas atividades resultam em alterações severas nos ecossistemas que compõem o bioma, especialmente a perda e a fragmentação de habitats (FARIA, 2016). O bioma Mata Atlântica exibe atualmente apenas 8,5\% dos remanescentes florestais acima de 100 hectares que já existiram (FUNDAÇÃO SOS MATA ATLÂNTICA, 2014).

A cidade de Porto Alegre apresenta $32 \%$ de sua cobertura original de Mata Atlântica (FUNDAÇÃO SOS MATA ATLÂNTICA, 2017) e é uma das capitais com maior porcentagem de remanescentes naturais do bioma. Nesse sentido, é imprescindível que haja o entendimento e o monitoramento da dinâmica dessas áreas florestais, visto que as ações de conservação e proteção dos remanescentes, bem como a recuperação e restauração de áreas perdidas, são grandes desafios (EISENLOHR et al., 2015).

A obtenção de informações sobre a dinâmica fenológica dos ecossistemas é fundamental no planejamento de ações que visem à conservação, pois permite maior sucesso dessas ações (KUPLICH et al., 2013). Uma forma de entender e monitorar a dinâmica de importantes áreas florestais é por meio do sensoriamento remoto, que parte do princípio de que cada objeto tem uma característica única de reflexão e emissão de energia eletromagnética, visto que a relação entre a radiação eletromagnética (REM) e a vegetação varia conforme as condições da vegetação, e é refletida e registrada em imagens (NOVO, 1992).

O sensoriamento remoto é amplamente aplicado para o monitoramento de coberturas vegetais, apresentando inúmeras aplicações ambientais e fazendo uso de sensores orbitais com as mais variadas características. A exemplo dos sensores com amplo campo de visada e menor resolução espacial, como o Satellite Pour l'Observation de la Terre - Vegetation (SPOT-VGT), que têm sido utilizados para monitorar regiões em maior escala (STROPPIANA et al., 2002). O satélite SPOT-VGT apresenta cobertura diária e probabilidade de imageamento livre de interferência de nuvens.

Dentre os produtos que são gerados a partir de técnicas de sensoriamento remoto destacam-se os índices de vegetação (ROUSE et al., 1974). Tradicionalmente, o monitoramento da vegetação por sensoriamento remoto tem sido realizado a partir desses índices, que são medidas radiométricas adimensionais que indicam a abundância relativa e a atividade de vegetação verde (JENSEN, 2009), permitindo a detecção de mudanças, no intuito de avaliar e monitorar a cobertura vegetal.

A relação existente entre a dinâmica espaço-temporal da vegetação e os índices de vegetação, atesta seu potencial em monitorar a cobertura vegetal sobre extensas áreas (SOUZA et al., 2016). Dessa forma, o Normalized Difference Vegetation Index (NDVI) é uma das técnicas mais utilizadas em sensoriamento remoto para estimar a quantidade de vegetação (ROUSE et al., 1974) e mudanças temporais em florestas. Isso porque apresenta forte relação com fatores climáticos e ambientais (KANG et al., 2014), possibilitando a verificação de alterações na fenologia e diminuição de biomassa a partir das variações no comportamento temporal/espectral da vegetação e alteração nos seus valores (WANG \& TENHUNEN, 2004; COSTA \& GUASSELLI, 2017).

Sabe-se que a fenologia estuda a ocorrência de eventos repetitivos e sua relação com mudanças no ambiente biótico e abiótico. Desse modo, identificar a fenologia em ciclos sazonais nas séries temporais de dados de vegetação permite observar anomalias e efeitos de mudanças climáticas nesses ciclos (KUPLICH et al., 2013). Através de imagens orbitais pode-se avaliar a dinâmica florestal e sua relação com as variáveis meteorológicas (KÄFER et al., 2018) visto que as imagens fornecem indicadores das variações das características do dossel vegetal (ROSA et al., 2013). Além disso, Leivas et al. (2014) comentam que é importante investigar a resposta temporal do NDVI em relação às variáveis climáticas em diferentes locais, já que um mesmo valor de NDVI pode estar associado à situação de seca em uma determinada região, e em outra não.

Sendo assim, o objetivo deste trabalho foi analisar a dinâmica espectral e temporal de remanescentes florestais pertencentes ao bioma Mata Atlântica, na cidade de Porto Alegre, por meio de séries temporais de NDVI provenientes de imagens SPOT-VGT (produto VGT-S10), no período de 2012-2014. Foram avaliadas principalmente as relações existentes entre a dinâmica dos remanescentes e as variáveis meteorológicas temperatura e precipitação.

\section{MATERIAL E MÉTODOS}




\section{Área de estudo}

A área de estudo compreende a cidade de Porto Alegre, capital do Estado do Rio Grande do Sul (Figura 1) que, junto com mais 13 municípios, compõe a região metropolitana de Porto Alegre (RMPA). A cidade possui 1.413.094 habitantes e uma área de 497 km² (IBGE, 2010). Além disso, apresenta uma geografia diversificada, com morros, planícies e um grande lago (o Guaíba). Seu clima é classificado como Cfa, ou seja, subtropical chuvoso com verões quentes e com precipitação média anual de 1.320,2 mm (MENGUE et al., 2017).
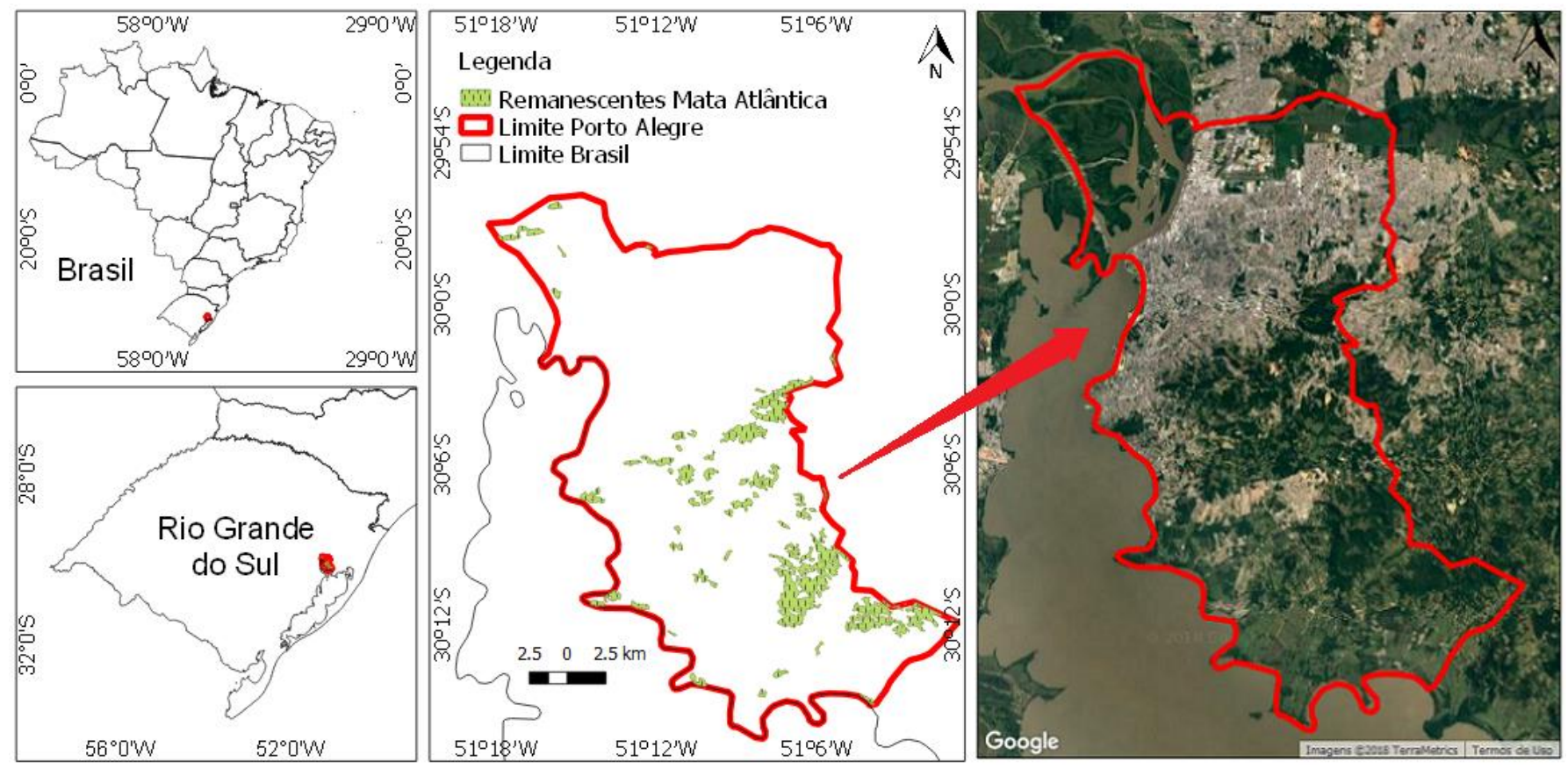

Figura 1. Localização da área de estudo no Brasil e Rio Grande do Sul (Fonte: adaptado de Google Earth).

De modo geral, o desmatamento no bioma Mata Atlântica no Estado do Rio Grande do Sul vem diminuindo. De acordo com dados do Instituto de Pesquisas Espaciais, apesar de terem sido reportadas áreas de desmatamento entre 2012-2013 e 2015-2016, a porcentagem de Mata, que são os de remanescentes florestais, ainda exibe 7,9\%, caindo de 8으 para 9o lugar no ranking de desmatamento dos estados nos últimos anos (FUNDAÇÃO SOS MATA ATLÂNTICA, 2014). Nesse contexto, é importante destacar que apesar de diversas fisionomias vegetacionais comporem o bioma Mata Atlântica, como as florestas ombrófilas (densa, aberta e mista) e as estacionais (semideciduais e deciduais) (MARMONTEL et al., 2013), os arquivos vetoriais utilizados neste estudo não contemplaram estas diferentes fisionomias nos remanescentes (FUNDAÇÃO SOS MATA ATLÂNTICA, 2014).

\section{Aquisição de dados e pré-processamento}

\section{Dados Spot Vegetation}

O sensor Vegetation (VGT) a bordo do satélite SPOT 4 foi lançado em abril de 1998, no intuito de fornecer imagens para o monitoramento da biosfera dos continentes em escala global com cobertura diária, com resolução espacial de $1 \mathrm{~km}^{2}$. (STROPPIANA et al., 2002). 0 sensor fornece produtos compostos, livres de interferências atmosféricas (CHAVES et al., 2014) e as informações a serem geradas estão relacionadas com a distribuição espacial e o estado da vegetação, com base em parâmetros biofísicos.

O VGT possui quatro bandas espectrais, cobrindo as faixas do visível, infravermelho próximo e infravermelho de ondas curtas, são elas: azul $(0,43-0,47 \mu \mathrm{m})$, vermelho $(0,61-$ $0,68 \mu \mathrm{m})$, infravermelho próximo $(0,78-0,89 \mu \mathrm{m})$ e infravermelho médio $(1,58-1,75 \mu \mathrm{m})$. Basicamente, os produtos VGT são fornecidos em três categorias: VGT - P (Primary), VGT-S (Synthesis) e VGT-D (MAISONGRANDE, 2004; CHAVES et al., 2014).

Os produtos VGT-S possuem maior aplicação na análise de mudanças no comportamento da vegetação, pois combinam dados diários, após realização da correção atmosférica e geométrica, de todos os segmentos de vegetação, com uso do algoritmo "maximum value composite" (MVC) (VICENTE et al., 2012), fornecendo sínteses de 10 dias de imagens com correções atmosféricas 
e gerando, posteriormente, uma imagem de NDVI com a menor interferência de nuvens possível (CHAVES et al., 2014; WOLTERS et al. 2016).

O NDVI é o índice sensível à presença de pigmentos que participam dos processos fotossintéticos, em particular ao conteúdo de clorofila (ROUSE et al., 1974) e proporciona o reconhecimento do início da estação de crescimento das plantas até a sua senescência (WANG \& TENHUNEN, 2004). O cálculo do NDVI, proposto por Rouse et al. (1973), é dado pela Equação 1:

$$
N D V I=\frac{(N I R-R E D)}{(N I R+R E D)}
$$

Em que NIR refere-se à reflectância no infravermelho próximo e RED refere-se à reflectância no vermelho.

Foram adquiridas 36 imagens decendiais por ano, entre o primeiro decêndio de janeiro de 2012 e o terceiro de maio de 2014, do produto NDVI disponíveis em: <http://free.vgt.vito.be/>, totalizando 87 imagens. Como os dados adquiridos são disponibilizados em formato RAR (Roshal ARchive), foi necessário descompactá-los por meio do software VGT Extract versão 1.4.1. Posteriormente as imagens foram convertidas para o formato do aplicativo ENVI.

Os dados originais de NDVI são disponibilizados em níveis de cinza (DN) na escala de 0 a 255, assim, os mesmos foram convertidos para que fossem trabalhados como uma grandeza física (NDVI). Para esta conversão, foi utilizada a Equação 2, proposta por Liu et al. (2010):

$$
\text { NDVI }=(\text { DN } \times 0,004)-0,1
$$

Em que NDVI refere-se ao índice de vegetação por diferença normalizada e DN aos níveis de cinza da imagem.

\section{Dados vetoriais}

Os dados vetoriais dos remanescentes do bioma Mata Atlântica utilizados neste estudo foram obtidos a partir da Fundação SOS Mata Atlântica, conforme mapeamento realizado em 2012 (FUNDAÇÃO SOS MATA ATLÂNTICA, 2012).

No estudo citado, o critério adotado para identificar as formações florestais naturais consistiu em mapear matas primárias e secundárias em estágios inicial, médio e avançado de regeneração. Neste caso, foram mapeadas as áreas de vegetação que possuem menor interferência antrópica e maior capacidade de proteger parte da sua biodiversidade original (FUNDAÇÃO SOS MATA ATLÂNTICA, 2014).

\section{Dados meteorológicos}

Os dados diários de precipitação e temperatura foram adquiridos da base de dados histórica proveniente do Instituto Nacional de Meteorologia (INMET, 2018), oriundos da estação meteorológica de Porto Alegre (Lat. 30,05으 S Long. 51,16으 O, altitude $47 \mathrm{~m}$ ). A fim de compatibilizar as análises, os dados de precipitação e temperatura foram integrados na mesma frequência de tempo das imagens de NDVI, isto é, com intervalos de 10 dias. Esses dados foram tabelados e estatísticas básicas foram calculadas.

\section{Análises}

As 87 imagens de NDVI foram empilhadas no software Envi 5.1 (1-JAN-2012 a 3-MAl-2014) e recortadas pelo arquivo vetorial limite da cidade de Porto Alegre. Em seguida, foi feito outro recorte pela máscara referente aos remanescentes florestais e foram calculadas as estatísticas (médias e desvios padrão) dos pixels de NDVI que pertenciam às áreas dos remanescentes.

A próxima etapa consistiu na integração dos dados pelo uso da ferramenta estatística OriginPro. A série temporal foi gerada e os resultados das relações entre as variáveis analisadas foram avaliados de forma gráfica.

Em última análise, foi testada a normalidade das séries de dados por meio do teste de Kolmogorov-Smirnov e, devido ao comportamento não normal das séries temporais de NDVI e variáveis climatológicas, foi realizada uma estatística pelo uso do coeficiente de correlação de Kendall (T) (a 95\% de probabilidade), que é uma medida de correlação não-paramétrica para dados ordinais. Uma classificação para esta correlação foi proposta por Callegari-Jacques (2003), e se dá de acordo com a Tabela 1.

Tabela 1. Avaliação qualitativa da correlação de Kendall.

\begin{tabular}{cccc}
\hline Classe & Positiva & Classe & Negativa \\
\hline 0 & Nula & 0 & Nula \\
0,0 a 0,3 & Fraca & 0,0 a $-0,3$ & Fraca \\
0,3 a 0,6 & Regular & $-0,3$ a $-0,6$ & Regular \\
0,6 a 0,9 & Forte & $-0,6$ a $-0,9$ & Forte \\
0,9 a 0,99 & Muito forte & $-0,9$ a $-0,99$ & Muito forte \\
1 & Plena & -1 & Plena \\
\hline Fonte: Callegari-Jacques (2003). & &
\end{tabular}

Este teste é capaz de avaliar a significância de uma tendência, baseando-se no ranqueamento dos dados. 
Desta maneira, os resultados variam entre -1 e 1 , onde é medido o grau de consistência crescente ou decrescente de uma tendência.

Valores próximos a 1 indicam tendência crescente, enquanto valores próximos a -1 indicam tendência decrescente. Os valores próximos a 0 indicam tendências inconsistentes ou não-tendência (MARIANO, 2015).

\section{RESULTADOS E DISCUSSÃO}

\section{Análise temporal do NDVI}

A análise da série temporal de NDVI de dados SPOTVGT 2012-2014 evidenciou a variação dos valores desse índice ao longo do tempo (Figura 2).

De modo geral, os valores de NDVI para toda a série estiveram no entorno de 0,7. De acordo com Costa e Guasselli (2017), valores do índice acima de 0,6 são valores característicos de áreas com média a elevada cobertura vegetal. O menor valor de NDVI encontrado neste estudo foi de 0,45 , no entanto, pode ser considerado um valor anômalo na série temporal, que ocorreu no último decêndio de setembro de 2013.

Nota-se que a série avaliada apresenta um padrão de menores valores de NDVI nos meses de inverno, e maiores nos meses de verão. Nesse contexto, Cordeiro et al. (2017), ao avaliarem diferentes grupos vegetais no Rio Grande do Sul, concluíram que este padrão ocorre porque em meses de verão a vegetação florestal se encontra com maior vigor, sendo os valores de NDVI maiores devido à maior disponibilidade de pigmentos fotossintetizantes.

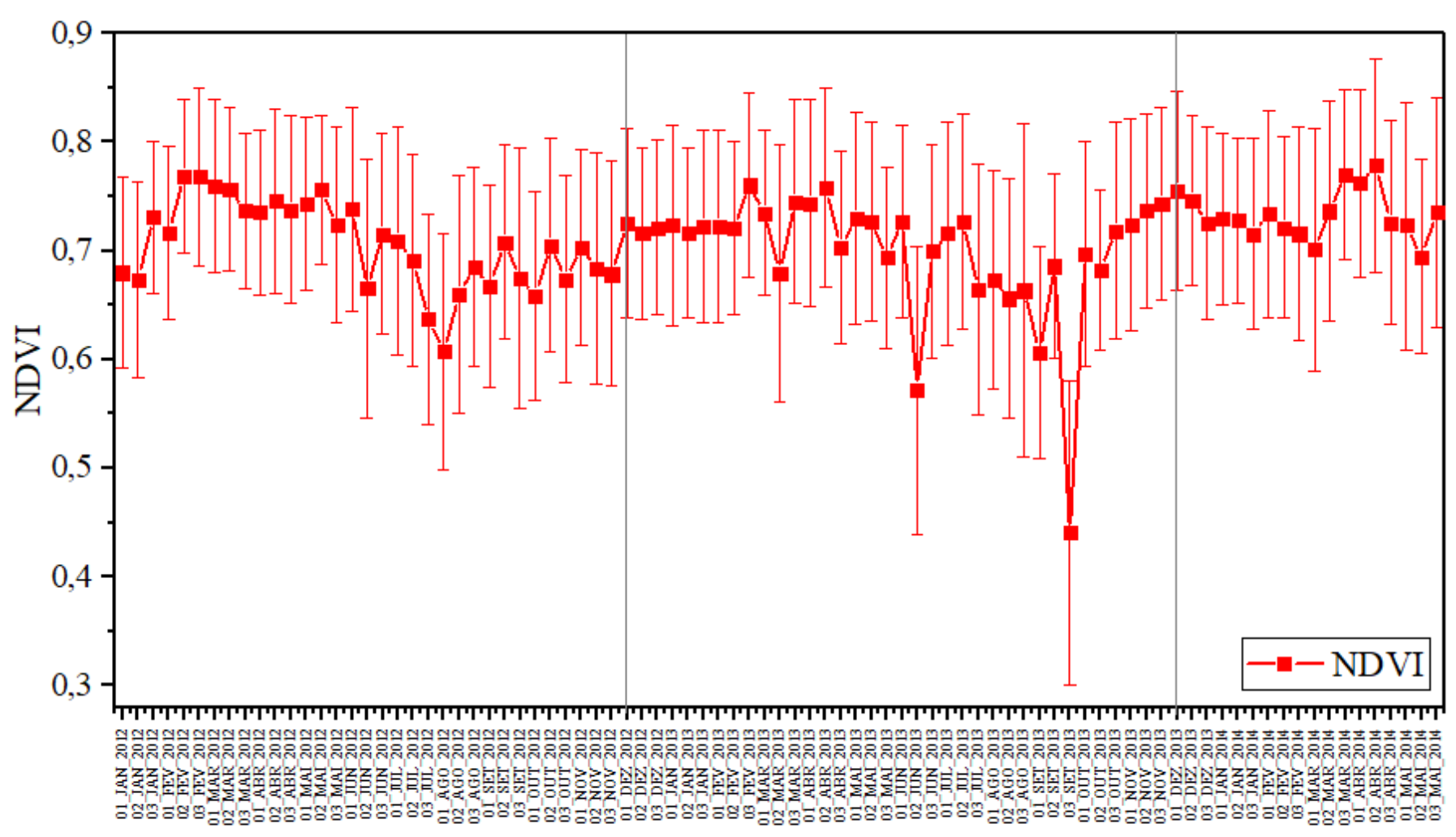

\section{Decêndio}

Figura 2. Distribuição temporal dos dados de NDVI dos remanescentes do bioma Mata Atlântica gerados a partir de composições decendiais do SPOT-VGT (2012-2014).

Em contrapartida no inverno, em fisionomias como Floresta Estacional Decidual ou Semidecidual, há perda de biomassa, reduzindo assim a oferta de pigmentos fotossintetizantes. Apesar de neste trabalho não terem sido consideradas fisionomias, é importante ressaltar que as Florestas Estacionais Semideciduais, caracterizadas pela perda de folhas de $20-50 \%$ no inverno, são as que ocorrem nas proximidades de Porto Alegre (LONGHI et al., 2008).

Barbosa et al. (2015) destacam que o NDVI é capaz de fornecer um vínculo claro entre a estrutura do ecossistema e as condições climáticas. Nesse sentido, apesar de a série temporal apresentar o padrão sazonal descrito, algumas variações intra-anuais são notadas nos ciclos de NDVI. Estas variações estão associadas às diferenças nas variáveis 
meteorológicas já que, segundo Wang et al. (2003), existe uma forte relação entre a temperatura, a precipitação e o NDVI. Esta relação fornece bases para predizer mudanças na vegetação que estão associadas às variações registradas na precipitação e na temperatura e serão comentadas neste artigo no tópico seguinte.

\section{Relação do NDVI com as variáveis climáticas}

A relação temporal entre NDVI e temperatura pode ser verificada na Figura 3. Observou-se que a resposta do índice de vegetação acompanhou a mudança na temperatura, ou seja, o decréscimo nos valores de NDVI nos meses de inverno ocorreram em função da resposta da vegetação à drástica diminuição da temperatura e vice- versa.

Quesada et al. (2017) comentaram que, no Sul do Brasil, o que determina a perda das folhas são as baixas temperaturas que precedem o inverno. Assim, como o NDVI é sensível ao aumento ou diminuição de biomassa verde, seus valores diminuem.

Resultado similar foi encontrado por Kuplich et al. (2013), ao avaliarem a relação entre o índice de vegetação EVI, a temperatura e a precipitação pluvial. Os autores comentam que a fenologia da vegetação e o ciclo de ganhos e perdas de massa foliar são controlados principalmente pela temperatura e pela disponibilidade hídrica.

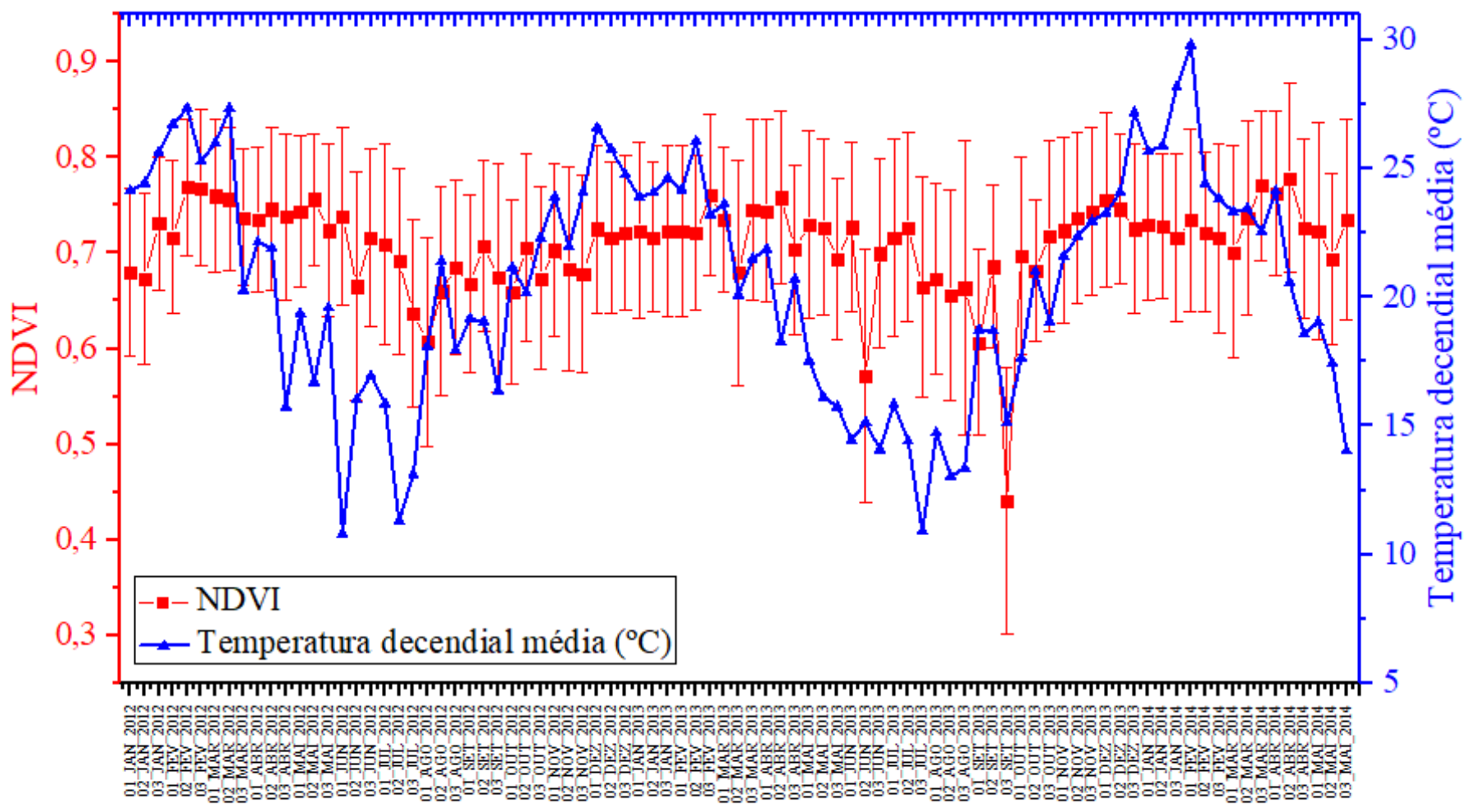

\section{Decêndio}

Figura 3. Distribuição temporal do NDVI e da temperatura decendial média (ำ). Os dados de temperatura apresentados foram obtidos a partir de médias decendiais de dados diários da estação meteorológica de Porto Alegre

Alguns autores relataram em seus trabalhos um atraso na resposta da floresta às mudanças na temperatura. Isso ocorre pelo fato de a vegetação responder após a mudança no ambiente e não durante (DALLA NORA \& SANTOS, 2010; QUESADA et al., 2017). Esta tendência explicada pode ser observada graficamente em vários pontos da série temporal, como por exemplo no segundo decêndio do mês de julho de 2012, em que a temperatura apresenta uma queda de mais de 4 으 em relação ao anterior, porém o valor de NDVI só apresenta uma baixa significativa 10 dias depois, onde passa de 0,69 para 0,63.

A análise da relação temporal existente entre o NDVI e a precipitação está representada na Figura 4. Os níveis de pluviosidade foram significativamente bem distribuídos durante o ano, porém de modo geral é possível observar que a maior quantidade de precipitação ocorre no segundo semestre do ano nos meses de agosto e setembro, enquanto as menores são observadas em abril, maio e 
junho.

Estes resultados concordam com o estudo de Domingues et al. (2015), que observaram na planície costeira do Rio Grande do Sul, um período de excesso hídrico de julho a outubro e outro de déficit hídrico de novembro a abril.

Como já comentado, os ciclos de aumento ou perda do vigor da vegetação são controlados principalmente pela temperatura e pela precipitação. No entanto, Segundo Kuplich et al. (2013), comparando estas variáveis entre si, a relação existente entre o índice de vegetação em questão (que representa o vigor da vegetação) e a temperatura, é mais forte do que a relação entre o vigor vegetativo e a precipitação pluvial.

O mesmo resultado foi observado neste estudo, em que o NDVI se demonstrou mais dependente de variações na temperatura do que na precipitação. Nessa perspectiva, Rosa et al. (2013) ao analisarem as relações entre os índices NDVI e EVI, variáveis meteorológicas, e índice de estiagem, concluíram que apenas uma seca prolongada poderia gerar um déficit hídrico que se manifestasse através da resposta espectral da floresta. Os autores comentam que isso ocorre porque há resiliência hídrica da floresta, isto é, a floresta possui capacidade de suportar curtos períodos de estiagem sem sofrer alterações visíveis no dossel.

Nesse mesmo contexto, Karamihalaki et al. (2016), em estudo similar utilizando os índices NDVI e NDWI de dados SPOT Vegetation, verificaram que as altas variações sazonais dos níveis de precipitação durante o ano têm maior efeito em ambos os índices, enquanto que uma distribuição anual da precipitação mais suave leva a menores correlações entre a precipitação e os dois índices.

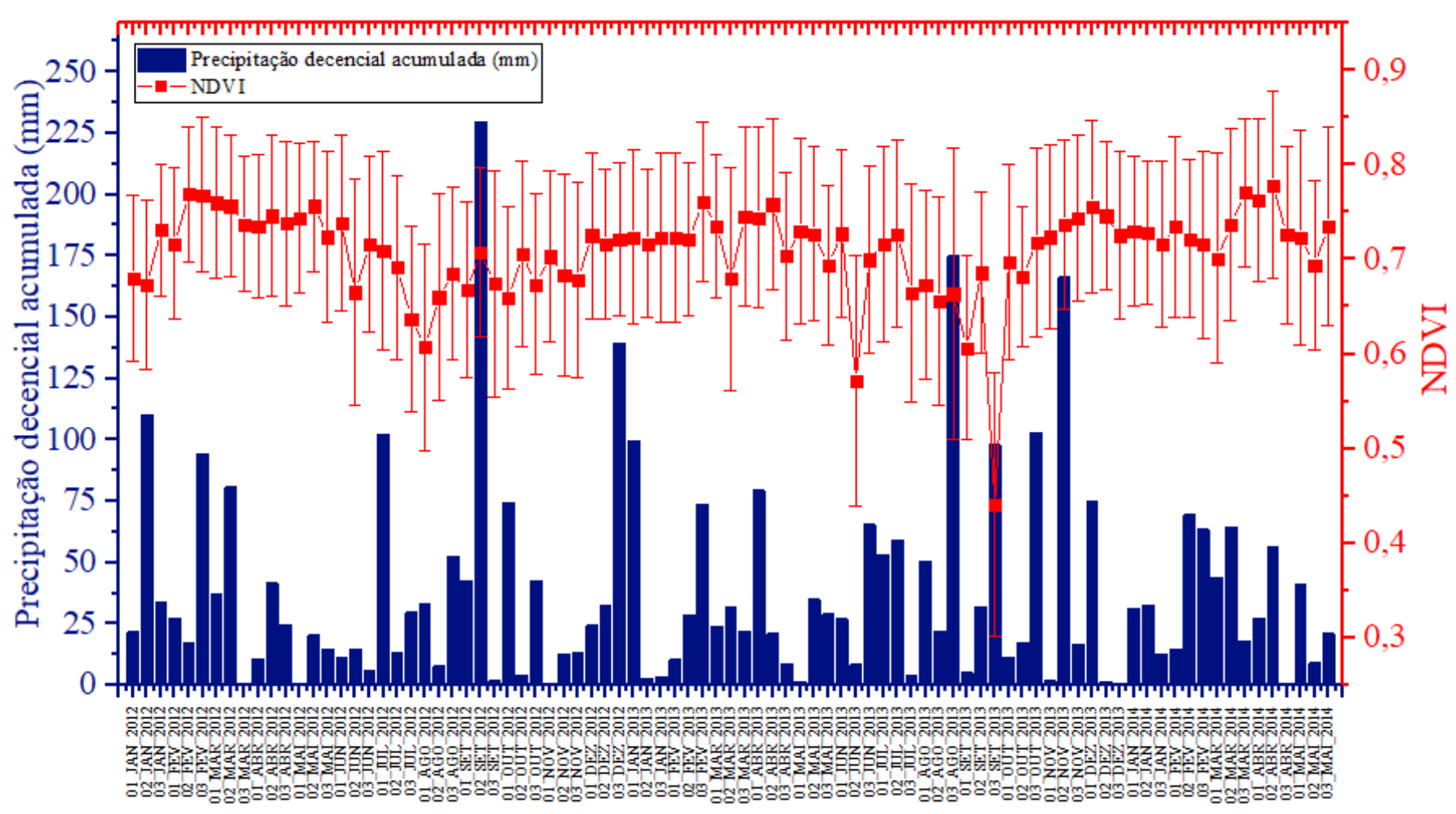

Decêndio

Figura 4. Distribuição temporal do NDVI e da precipitação decendial acumulada $(\mathrm{mm})$. Os dados de precipitação acumulada apresentados foram obtidos a partir da soma de dados diários da estação meteorológica de Porto Alegre.

A fim de analisar estatisticamente a existência de correlação entre as variáveis estudadas neste trabalho, foi realizado o teste de correlação de Kendall (T). Os resultados podem ser vistos na Tabela 2.

Após a aplicação do teste, observou-se estatisticamente que a variável temperatura apresenta as maiores correlações com o índice de vegetação NDVI. Estas são classificadas como regulares positivas (CALLEGARIJACQUES, 2003), apresentando valores de 0,33 e 0,47 e um e dois meses de defasagem, respectivamente. Em outras palavras, uma variável aumenta em função da outra e a temperatura leva entre um e dois meses para causar diferenças espectrais significativas no NDVI. Por outro lado, a precipitação apresentou correlação negativa 
classificada como regular negativa $(-0,34)$ e seu maior valor ocorreu com um mês de defasagem. Este tipo de resultado de correlação inversa demonstra que enquanto uma variável aumenta, ocorre decréscimo da outra.

Tabela 2. Correlação entre as variáveis meteorológicas e o NDVI pelo teste de correlação de Kendall (*Refere-se a 95\% de significância).

\begin{tabular}{ccc}
\hline Defasagem (meses) & Temperatura & Precipitação \\
\hline 1 & $0,33^{*}$ & $-0,34^{*}$ \\
2 & $0,47^{*}$ & $-0,20$ \\
3 & 0,31 & $-0,04$ \\
4 & 0,13 & 0,09 \\
5 & 0,07 & 0,06 \\
\hline
\end{tabular}

A existência de correlações fortes entre NDVI e precipitação para a região sul não são tão significativas quanto em outros locais onde há, por exemplo, períodos mais frequentes de estiagem. Por fim, a variação sazonal do NDVI dos remanescentes pode ser vista espacialmente na Figura 5.

Segundo a classificação climática do estado do Rio Grande do sul, as estações são bem definidas com invernos rigorosos e verões quentes, o que faz com que a temperatura seja um fator mais influente no desenvolvimento da vegetação do que a precipitação (DALLA NORA et al., 2010).

As maiores variações sazonais para cada ano são representadas pelas imagens que exibiram os maiores (verão) e menores (inverno) valores médios de índice de vegetação. Como continuidade do estudo, sugere-se que mais anos sejam considerados na série temporal, no intuito de traçar a dinâmica dos remanescentes captando uma maior quantidade de variações temporais possíveis nas variáveis analisadas.

\section{CONCLUSÕES}

A utilização de uma série temporal de dados SPOT-VGT (2012-2014) foi eficaz na obtenção de informações sobre a fenologia da vegetação de remanescentes do bioma Mata Atlântica na cidade de Porto Alegre, principalmente em função de sua alta resolução temporal.

O padrão de comportamento da vegetação apresentou um ciclo definido nos dados decendiais de NDVI e este ciclo está claramente associado a variações nas variáveis climáticas temperatura e precipitação.

As variações no NDVI podem ajudar a compreender o efeito de fatores climáticos sobre a vegetação local, e pode auxiliar no planejamento e mitigação de grande escala.

A temperatura mostrou-se mais relacionada com os dados de NDVI do que a precipitação, principalmente pela capacidade dos remanescentes florestais de resistirem a curtos períodos de déficit hídrico.
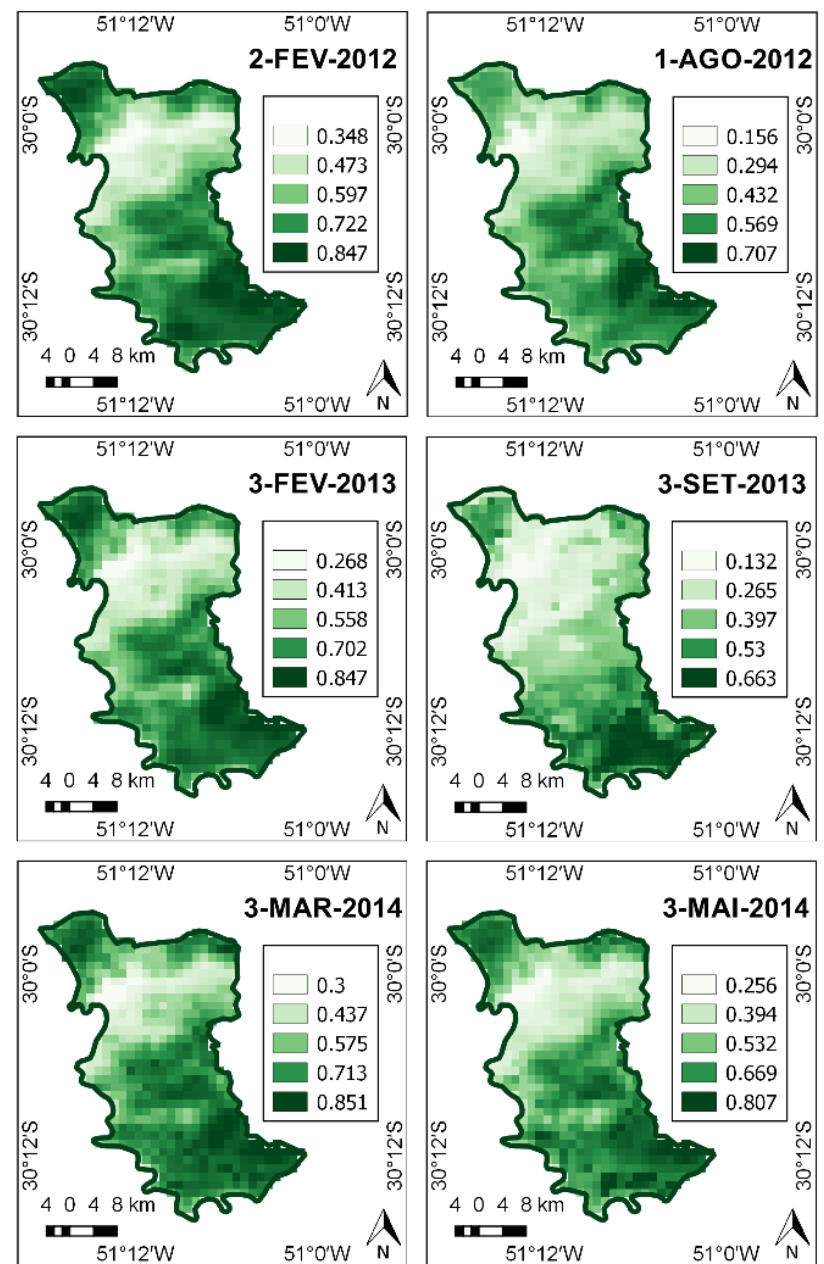

Figura 5. Avaliação espacial sazonal do NDVI. Imagens dos decêndios correspondentes aos maiores e menores valores de NDVI dentro de cada ano foram escolhidas para a representação.

\section{AGRADECIMENTOS}

Os autores agradecem à Coordenação de Aperfeiçoamento de Pessoal de Nível Superior (CAPES) e ao Conselho Nacional de Desenvolvimento Científico e Tecnológico (CNPq) pelo apoio financeiro. Os dados SPOT Vegetation são cortesia do Flemish Institute for Technological Research (VITO). Ainda, os autores agradecem ao Instituto Nacional de Meteorologia pelos dados meteorológicos e ao Instituto de Pesquisas Espaciais pelos dados vetoriais utilizados nesta pesquisa. 


\section{REFERÊNCIAS}

BARBOSA, H.A. et al. Recent trends in vegetation dynamics in the South America and their relationship to rainfall. Natural Hazards, v.77, n.2, p.883-899, 2015.

CALLEGARI-JACQUES, S.M. Bioestatística: princípios e aplicações. Porto Alegre: Artmed, 2003

CHAVES, M.E.D. et al. Uso da modelagem estatística para monitoramento da vegetação no Parque Nacional da Serra da Canastra, Minas Gerais. Caderno de Geografia, v.24, n.1, p.120132, 2014.

CORDEIRO, A.P. et al. Regiões homogêneas de vegetação utilizando a variabilidade do NDVI. Ciência Florestal, v.27, n.3, p.883-896, 2017.

COSTA, R.C.; FUTEMMA, C.R.T. Racionalidade com compromisso: os assentados do Ribeirão Bonito (Teodoro Sampaio-SP) e o Projeto de Conservação Ambiental. Ambiente \& Sociedade, v.9, p.128-148, 2006.

COSTA, L.C.B.; GUASSELLI, L.A. Dinâmica sazonal de remanescentes da mata atlântica, a partir de séries temporais NDVI/MODIS. Geo UERJ, n.30, p.214-239, 2017.

CYSNEIROS, V.C.; BRAZ, D.M.; PELISSARI, A.L.; SANTOS, K.S.M. Composição Florística e Fitogeografica de uma Floresta Atlântica no Sudeste Brasileiro. BIOFIX Scientific Journal, v.1, n.1, p.98106, 2016.

DALLA NORA, E.L.; SANTOS, J.E. Análise da dinâmica sazonal de duas formações florestais do bioma Mata Atlântica com base em índice de vegetação. Perspectiva, v.34, n.125, p.41-51, 2010.

DOMINGUES, A.L. et al. Delimitação da área de preservação permanente da Lagoa dos Gateados, na planície costeira do Rio Grande Do Sul (RS), utilizando séries de imagens de satélite e dados hidrológicos históricos. Revista Brasileira de Geografia Física, v.8, n.3, p.776-792, 2015.

EISENLOHR, P.V. et al. The Brazilian Atlantic Forest: new findings, challenges and prospects in a shrinking hotspot. Biodiversity and Conservation, v.24, n.9, p.2129-2133, 2015.

FARIA, C.O. Governança da biodiversidade sob a perspectiva de sistemas sócio-ecológicos: o caso do bioma Mata Atlântica. 2016. 126p. (Tese de doutorado).

FUNDAÇÃO SOS MATA ATLÂNTICA. Atlas remanescentes. 2012. Disponível em: http://mapas.sosma.org.br/dados

FUNDAÇÃO SOS MATA ATLÂNTICA. Nossas causas. 2014. Disponível em: https://www.sosma.org.br/nossas-causas/mataatlantica/

FUNDAÇÃO SOS MATA ATLÂNTICA. Atlas dos remanescentes florestais da Mata Atlântica: período 2015-2016. São Paulo, 2017. Disponível em: https://www.sosma.org.br/wpcontent/uploads/2015/11/Atlas-munic\%C3\%ADpios-SOS-RioGrande-do-Sul-OK.pdf

IBGE - INSTITUTO BRASILEIRO DE GEOGRAFIA E ESTATÍSTICA.
2010. Sistema IBGE de recuperação automática: banco de dados agregados. Rio de Janeiro: IBGE, 2010. Disponível em: http://www.sidra. ibge.gov.br

JENSEN, J.R. Sensoriamento remoto do ambiente: uma perspectiva em recursos terrestres. 2.ed. São José dos Campos: Parêntese, 2009.

KÄFER, P.S. et al. Modelling Pinus elliottii growth with multitemporal Landsat data: a study case in Southern Brazil, Boletim de Ciências Geodésicas, v.24, p.286-299, 2018.

KANG, L. et al. Use of Geographically Weighted Regression Model for Exploring Spatial Patterns and Local Factors Behind NDVIPrecipitation Correlation. IEEE Journal of selected topics in applied earth observations and remote sensing, v.7, n.11, p.4530-4538, 2014.

KARAMIHALAKI, M. et al. Monitoring Drought Effects on Mediterranean conifer forests using SPOT-VEGETATION NDVI and NDWI time series. ESA Living Planet, 2016.

KUPLICH, T.M. et al. Série temporal de índice de vegetação sobre diferentes tipologias vegetais no Rio Grande do Sul. Revista Brasileira de Engenharia Agrícola e Ambiental, n.10, p.11161123, 2013.

LEIVAS, J.F. et al. Monitoramento da seca $2011 / 2012$ no nordeste brasileiro a partir do satélite SPOT-Vegetation e TRMM Engenharia na agricultura, v.22, n.3, p.211-221 2014.

LIU, S. et al. Vegetation change based on SPOT-VGT data from 1998-2007, northern China. Environmental Earth Sciences, v.60, p.1459-1466, 2010.

LONGHI S.J. et al. Caracterização fitossociológica do estrato arbóreo. Ciência Rural, v.38, n.6, p.1630-1638, 2008.

MAISONGRANDE, P. et al. VEGETATION/SPOT: An operational mission for the Earth monitoring; presentation of new standard products. International Journal of Remote Sensing, v.25, n.1, p.914, 2004.

MARIANO, D.A. Detecção e avaliação de seca agronômica através da análise de séries temporais de dados MODIS e Persiann. 2015. 112p. (Dissertação de mestrado).

MARMONTEL, C.V.F. et al. Caracterização da vegetação secundária do bioma mata atlântica com base em sua posição na paisagem. Bioscience Journal, v.29, n.6, p.2042-2052, 2013.

MENGUE, $V$. et al. Análise da expansão urbana em áreas suscetíveis à inundação utilizando o modelo HAND: o caso da Região Metropolitana de Porto Alegre, Brasil. Revista de Geografia e Ordenamento do Território, n.12, p.231-253, 2017.

NOVO, E.M.L.M. Sensoriamento Remoto: princípios e aplicações. São Paulo: Edgard Blücher, 1992.

QUESADA, H.B. et al. Análise da vegetação ripária em bacia hidrográfica utilizando índice de vegetação normalizada (NDVI) no município de Maringá-PR. GEO UERJ, n.31, p.439-455 2017.

ROSA, P.A. et al. Dinâmica da floresta do Parque Estadual do 
Turvo com índices de vegetação. Floresta e Ambiente, v.20, n.4, p.487-499, 2013.

ROUSE, J.W. et al. Monitoring the vernal advancement and retrogradation (green wave effect) of natural vegetation. Progress Report RSC 1978-2. College Station: Texas \& A.M. University, 1974.

SILVA, L.G. et al. Fragmentação da Mata Atlântica de interior: análise de paisagem do corredor verde Sul-Americano e florestas do Alto Paraná. Boletim de Geografia, v.32, n.3, p.61-68, 2014.

STROPPIANA, D. et al. Radiometric analysis of SPOT-Vegetation images for burnt area detection in Northern Australia. Remote Sensing of Environment, v.82, n.1, p.21-37, 2002.

SOUZA, G.M. et al. Análise sazonal da vegetação do cerrado por meio de dados do sensor MODIS no distrito federal (BRASIL). Boletim Goiano de Geografia, v.36, n.3, p.502-520, 2016.

TEIXEIRA, G.M.; FIGUEIREDO, P.H.A.; FERRAZ, S.F.B.; SALEMI, L.F.; RANZINI, M.; RIZZI, N.E. Análise de Classificadores de Estágios Sucessionais em um Fragmento de Mata Atlântica. BIOFIX Scientific Journal, v.4, n.2, p.88-96, 2019.

VICENTE, L.E. et al. Séries temporais de NDVI do sensor SPOT Vegetation e algoritmo SAM aplicados ao mapeamento de cana-de-açúcar. Pesquisa Agropecuária Brasileira, v.47, n.9, p.1337-1345, 2012.

WAGNER, A.P.L. et al. Tendências temporais de índices de vegetação nos campos do Pampa do Brasil e do Uruguai. Pesquisa agropecuária brasileira, v.48, n.9, p.1192-1200, 2013.

WANG, J. et al. Temporal responses of NDVI to precipitation and temperature in the central Great Plains, USA. International Journal of Remote Sensing, v.24, n.11, p.2345-2364, 2003.

WANG, Q.; TENHUNEN, J.D. Vegetation mapping with multitemporal NDVI in North Eastern China Transect (NECT). International Journal of Applied Earth Observation and Geoinformation, v.6, n.1, p.17-31, 2004.

WOLTERS, E.; SWINNEN, E.; TOTÉ, C.; STERCKX, S. SPOT-VGT Collection 3 Products User Manual. 2016. Disponível em: http://www.spot-

vegetation.com/pages/SPOT_VGT_PUM_v1.0.pdf

Recebido em 03-06-2019 Aceito em 04-07-2019 